\title{
Systematic review, meta-analysis and statistical analysis of laparoscopic supracervical hysterectomy vs. endometrial ablation
}

\author{
(D) Greg J. Marchand ${ }^{1}$, (D) Ali Azadi2 ${ }^{2}$, (D) Katelyn Sainz ${ }^{1,3}$, (D) Ahmed Masoud ${ }^{1}$, (D) Sienna Anderson ${ }^{1}$, (D) Stacy Ruther ${ }^{1}$, \\ (D) Kelly Ware1,4, (D) Sophia Hopewell ${ }^{1}$, (D) Giovanna Brazil1 ${ }^{1}$, (D) Alexa King1 ${ }^{1}$, (D) Jannelle Vallejo 1,3, \\ (D) Kaitlynne Cieminski1 1 (D) Anthony Galitsky1 ${ }^{1}$, (D) Allison Steele ${ }^{4,5}$, (D) Jennifer Love4,5 \\ 1Department of Minimally Invasive Surgery, The Marchand Institute for Minimally Invasive Surgery, Mesa, United States of \\ America \\ 2Department of Urogynecology, Star Urogynecology Advanced Pelvic Health Institute for Women, Arizona, United States of \\ America \\ 3Washington University of Health and Science School of Medicine, San Pedro, Belize \\ ${ }^{4}$ International University of Health Sciences School of Medicine, Basseterre, Saint Kitts and Nevis \\ 5 Midwestern University School of Medicine, Arizona, United States of America
}

\section{Abstract}

Objective: This meta-analysis aimed to compare the effect of laparoscopic supracervical hysterectomy (LSH) with endometrial ablation (EA) in terms of general and menstrual-related quality of life in women opting for surgical treatment for abnormal uterine bleeding.

Material and Methods: Sources searched included PubMed, Cochrane library, Scopus, and Web of Science for relevant clinical trials. Main outcomes of interest included: quality of life assessed using medical outcomes survey short form-36 (SF-36), (SF-12), operation time, time from operation to discharge, pain, fever, and hemoglobin level. Screening and data extraction were performed independently and the analysis was conducted using Review Manager Software v5.4.1.

Results: Four clinical trials were included. Results of SF-12 score showed that there was no significant difference between the LSH and EA groups for either mental or physical component score overall mean difference (MD): $-4.15(-16.01,7.71 ; \mathrm{p}=0.49)$ and MD: 2.67 (-0.37, 5.71; $\mathrm{p}=0.08$ ), respectively. Subgroup analysis of the SF-36 showed that only two components, general health and social function, were significantly improved in the LSH group ( $\mathrm{p}<0.01)$ while the other six sub-scores did not differ between groups. The overall MD significantly favored the EA group for: operation time [MD: $72.65(35.48,109.82 ; \mathrm{p}=0.0001)]$, time from operation to discharge [MD: 13.61 (3.21, 24.01; $\mathrm{p}=0.01)]$, hemoglobin level outcome [MD: $0.57(0.40,0.74) ; \mathrm{p}<0.01$ ], and pain score [standardized MD: $0.46(0.32,0.60 ; \mathrm{p}<0.01)]$.

Conclusion: LSH has better outcomes for quality of life. This includes patient indicated responses to social health, general health, and superior hemoglobin levels at all measured points postoperatively. EA, however, was consistently associated with less operative time, a shorter hospital stay and is also considered by the authors to be a more minimally invasive technique which can also result in satisfying outcomes. ( $\mathrm{J}$ Turk Ger Gynecol Assoc 2021; 22: 97-106)

Keywords: Supracervical, supracervical hysterectomy, endometrial ablation, abnormal uterine bleeding, systematic review

Received: 13 October, 2020 Accepted: 14 January, 2021

This Manuscript has been reviewed by the Institutional IRB Board at Marchand Institute and was found to be exempt from IRB review (September, 2020).

Address for Correspondence: Greg J. Marchand

e.mail: gm@marchandinstitute.org ORCID: orcid.org/0000-0003-4724-9148

${ }^{\circ}$ Copyright 2021 by the Turkish-German Gynecological Education and Research Foundation - Available online at www.jtgga.org

Journal of the Turkish-German Gynecological Association published by Galenos Publishing House.

DOI: 10.4274/jtgga.galenos.2021.2020.0185 


\section{Introduction}

Abnormal uterine bleeding (AUB) is one of the most common gynecological problems that affects a quarter of women in the United States (1). It significantly impairs the quality of life of many women and accounts for a fifth of all hospital gynecology referrals (2). Menorrhagia is subjectively defined by some authors as excessive cyclic menstrual bleeding occurring over several consecutive cycles (3) and is objectively defined as a menstrual blood loss of $80 \mathrm{~mL}$ or more per menstrual cycle by other authors (4). Only $34 \%$ of females complaining of menorrhagia reported bleeding of more than $80 \mathrm{~mL}$ (5). Therefore, there is a discrepancy between women's perception of their menstrual bleeding and objective measures of the amount of blood loss (6). Endometrial polyps, adenomyosis, and fibroid(s) are common structural abnormalities of the uterus, which are associated with menorrhagia $(7,8)$. AUB can also result from an abnormality of the endometrium (hyperplasia or malignancy), a disorder of ovulation, and coagulation defects (9).

Generally, a medical approach is used as the first line for the treatment of AUB, but it has a comparably high failure rate. About $42 \%$ of women using an intrauterine hormonal system and $77 \%$ of those on oral drugs will need to undergo surgical treatment within five years (10). The two most common surgical approaches for the treatment of AUB are hysterectomy, where the uterus is removed, and endometrial ablation (EA), where the endometrium is thermally destroyed with uterine preservation (11). Abdominal hysterectomy had been the only definitive treatment for AUB, until the development of endoscopy introduced laparoscopic hysterectomy and hysteroscopic EA (12). Most women prefer the laparoscopic approach because it has a less invasive nature and a shorter hospital stay (13). Laparoscopic supracervical hysterectomy (LSH) only removes the uterine body, which is the main source of AUB (14). This is also sometimes referred to as subtotal or partial hysterectomy. In many cases, this procedure offers a less complex surgery that avoids difficult surgical dissection around the cervix and bladder (15). Although controversial, many authors have suggested leaving the cervix and its ligaments can reduce the risk of vaginal vault prolapse, and most authors agree it can reduce the risk of ureteric injury in some cases (16). The availability of newer surgical equipment and the improvements in laparoscopic training have simplified LSH.

EA is an effective and minimally invasive surgical technique that has been developed to remove the entire thickness of the endometrium while sparing the uterine body $(17,18)$. After EA, any residual endometrium is theoretically beneath the created scar, and as a result this prevents further bleeding. Although it improves the general and menstrual-related quality of life, about $20 \%$ of women who have this treatment will require a hysterectomy for relief of their symptoms at some point (19). In this meta-analysis, the aim was to compare the effect of LSH with EA in terms of general and menstrual-related quality of life in women opting for surgical treatment for AUB.

\section{Material and Methods}

This systematic review and meta-analysis was performed following the Preferred Reporting Items for Systematic Reviews and Meta-Analyses (PRISMA) statement (20) and the guidelines reported in the Cochrane Handbook for Systematic Reviews of Interventions (21). Informed consent was not applicable to this study as it is a systematic review performed according to the PRISMA statement protocol.

\section{Search strategy}

Online databases which were searched were: Web of Science, Scopus, Cochrane Central, and PubMed, without any restrictions on time or languages. We used the keywords: "ablation", "hysterectomy", "laparoscopy*", and "bleeding" and combined these words by "AND" or "OR" as appropriate for the search.

\section{Eligibility criteria}

All studies were included that met the following criteria: 1) patients - women with AUB; 2) intervention - $\mathrm{LSH}$; 3) comparator - EA or resection; 4) outcomes - quality of life assessed using medical outcomes survey short form 36 (SF-36), SF-12, operation time, time from operation to discharge, pain, fever, and hemoglobin level; and 5) study design - only randomized clinical trials (RCTs) were included. Studies were excluded if they contained any of the following criteria: 1) non-RCTs; 2) patients treated with hysterectomy using other techniques; 3) single-armed trials, or with different comparators other than EA or resection; 4) animal trials; and 5) studies that have no available full-text.

\section{Screening of search results}

The results of the search were exported into Endnote X8.0.1 (Build 1044), with the removal of duplicates automatically by computer. After that, the studies were screened manually in two steps. Firstly, title and abstract screening. Secondly, fulltext screening of the studies which had passed the first step. Articles were included on the basis of the inclusion criteria as defined earlier and studies were removed studies that didn't meet these criteria.

\section{Data extraction and analysis}

After the screening step, data were extracted from the selected studies and the outcomes were categorized into two 
main groups: 1) baseline and demographic data of patients in each study, including age, body mass index (BMI) (kg/ $\mathrm{m}^{2}$ ), parity, preoperative hemoglobin level, and incidence of dysmenorrhea; 2) outcomes including quality of life assessed using the medical outcomes SF-36, which consists of eight components (general health, physical function, limitations on life functions (physical), limitations on life functions (emotional), mental health, social function, vitality, and pain), SF-12 which consists of two components [physical component summary (PCS) and mental component summary (MCS)], operation time, time from operation to discharge, pain, fever, and hemoglobin level.

\section{Statistical analysis}

We used Review Manager Software (RevMan 5.4.1) to perform our analysis. We expressed dichotomous outcomes using percent and total, while continuous outcomes were expressed using mean difference (MD) and standard deviations, relative to $95 \%$ confidence interval. In the case of outcomes reported by different tools or parameters, we used the standardized MD in the analysis. Heterogeneity was assessed using statistical $\mathrm{I}^{2}$ test and $\mathrm{p}$-value of the chi-square test, where outcomes with $\mathrm{I}^{2}>50 \%, \mathrm{p}<0.1$ were considered heterogeneous, while outcomes with $\mathrm{I}^{2}<50 \%, \mathrm{p}>0.1$ were considered homogeneous. The homogenous data was analyzed using a fixed-effects model, while heterogeneous outcomes were analyzed using the random-effects model.

\section{Quality assessment}

The quality of this systematic review and meta-analysis was evaluated using the Grading of Recommendations Assessment, Development and Evaluation (GRADE) guidelines according to the Cochrane risk of bias (ROB) tool for clinical trials (22). The ROB was performed for the included studies. The Cochrane ROB assessment tool includes the following domains: random sequence generation (selection bias), allocation sequence concealment (selection bias), blinding of participants and personnel (performance bias), blinding of outcome assessment (detection bias), incomplete outcome data (attrition bias), selective outcome reporting (reporting bias) and other potential sources of bias. The authors' judgment was categorized as "low risk", "high risk", or "unclear risk" of bias.

\section{Results}

\section{Summary of included studies}

The literature search and references retrieved 127 studies. Twenty studies met the study criteria and were included in full-text screening. Four of these studies met the full eligibility criteria after full-text screening and were included in the metaanalysis. Figure 1 illustrates the PRISMA statement for the literature search. The four acceptable studies included 1018 patients for analysis (23-26), treated by either LSH or EA or resection - grouped as EA hereafter. A total of 517 underwent LSH, while 501 patients underwent EA. The mean age of patients in the LSH group was $47.2+4.77$ years, while that of the EA group was $47.55+4.6$ years. Table 1 shows a detailed summary of the included participants, their demographic characteristics, BMI, parity, preoperative hemoglobin, and the number of patients who suffered from dysmenorrhea.

\section{Results of risk of bias assessment}

The ROB assessment result yielded an overall moderate risk, according to Cochrane's tool (21). Regarding randomization, all studies were at low risk of randomization (23-26). As for allocation concealment, two studies reported insufficient details; therefore, they were categorized as "unclear risk" $(25,26)$. Sesti et al. (24) reported adequate allocation concealment, so it was considered low risk while the study of Cooper et al. (23) showed inadequate concealment of allocation, so it was marked as high risk. Concerning blinding of participants and personnel, all studies did not report sufficient details about blinding of participants and personnel, so they were designated unclear risk, except Sesti et al. (24) that

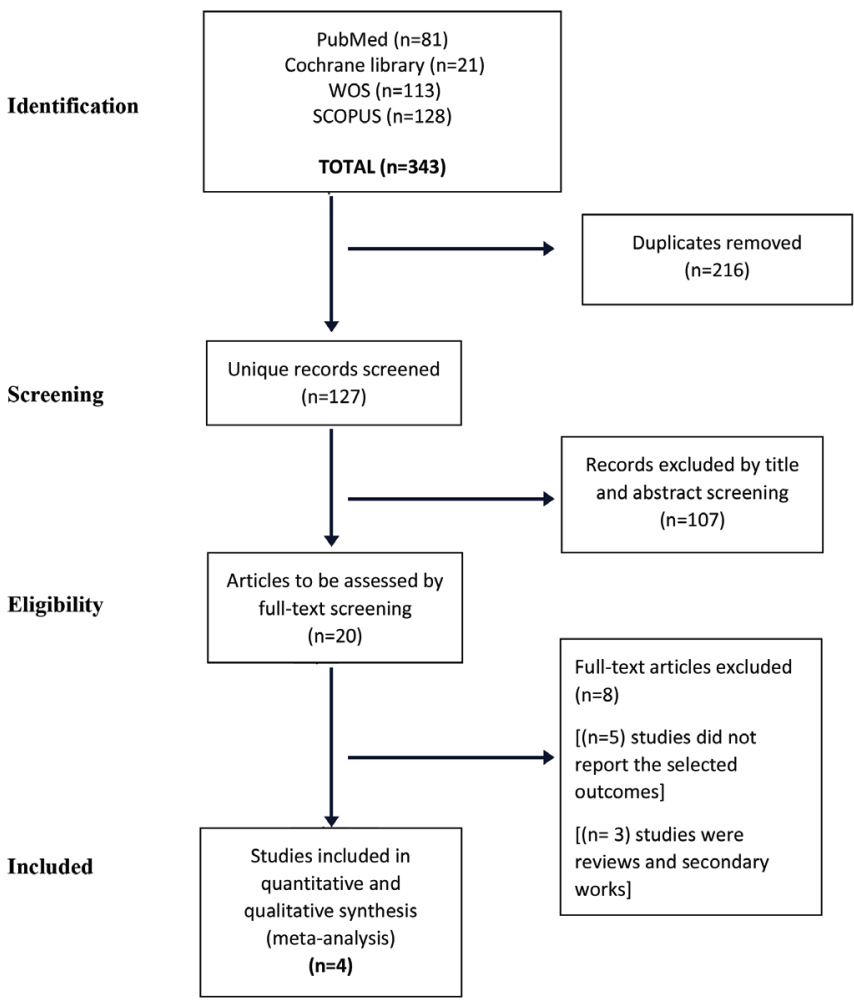

Figure 1. Shows the PRISMA statement of our literature search

PRISMA: Preferred reporting Items for systematic reviews and meta-analyses, WOS: Web of Science 


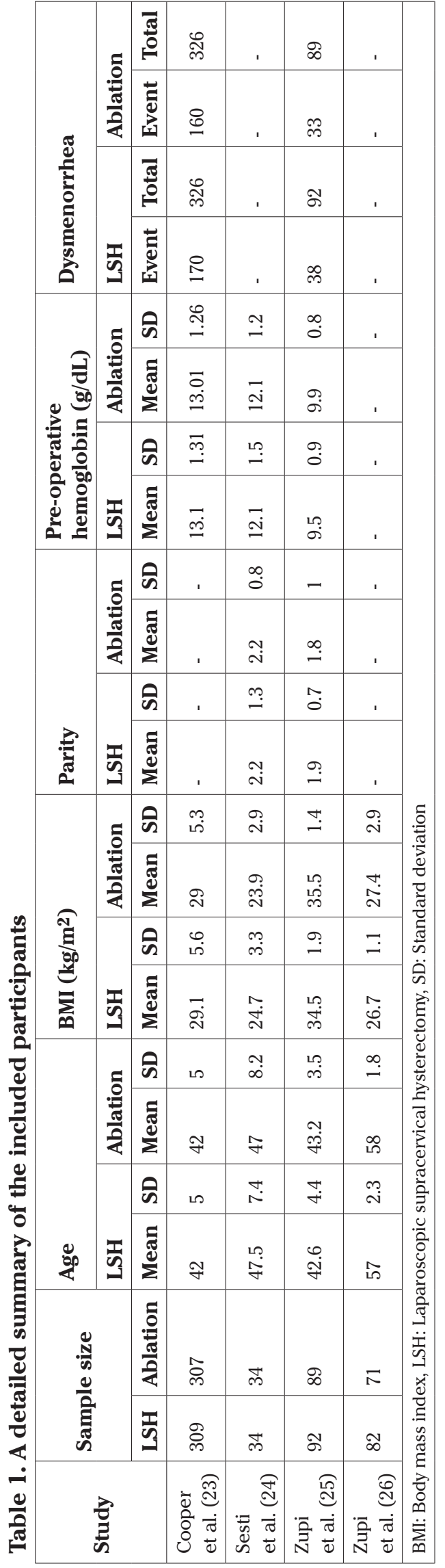

reported adequate blinding of participants and personnel, so it was categorized as low risk. As for blinding of outcome assessment, blinding was likely effective in all studies, so they were at low risk (23-26). All studies were at low risk of attrition bias. Moreover, all studies were at low risk of selective reporting. There was no other bias in all studies except for Cooper et al. (23) with unclear risk. A detailed illustration of the ROB of included trials is summarized in Figure 2.

\section{Analysis of outcomes}

\section{Short form survey-12}

Two studies reported the SF-12 outcome $(23,26)$. SF-12 consists of a PCS and a MCS. Regarding MCS, the overall mean difference showed that there was no significant favoring of either group over the other [MD: -4.15 (-16.01, 7.71), $(p=0.49)$ ], Pooled data was heterogeneous ( $<<0.01) ; I^{2}: 99 \%$. Regarding PCS, there was no significant difference between both groups [MD: 2.67 (-0.37, 5.71), $(\mathrm{p}=0.08)$ ]. Pooled analysis was heterogeneous $(\mathrm{p}=0.001) ; \mathrm{I}^{2}: 91 \%$ as shown in Figure 3. Heterogeneity was not resolvable in either component, either by the leave-one-out method or subgroup analysis because only two studies had reported the outcome.

\section{Survey short form-36}

SF-36 outcome was reported by two studies $(24,25)$. SF-36 consists of eight components (general health, physical function, limitation on life functions (physical), limitation on life functions (emotional), mental health, social

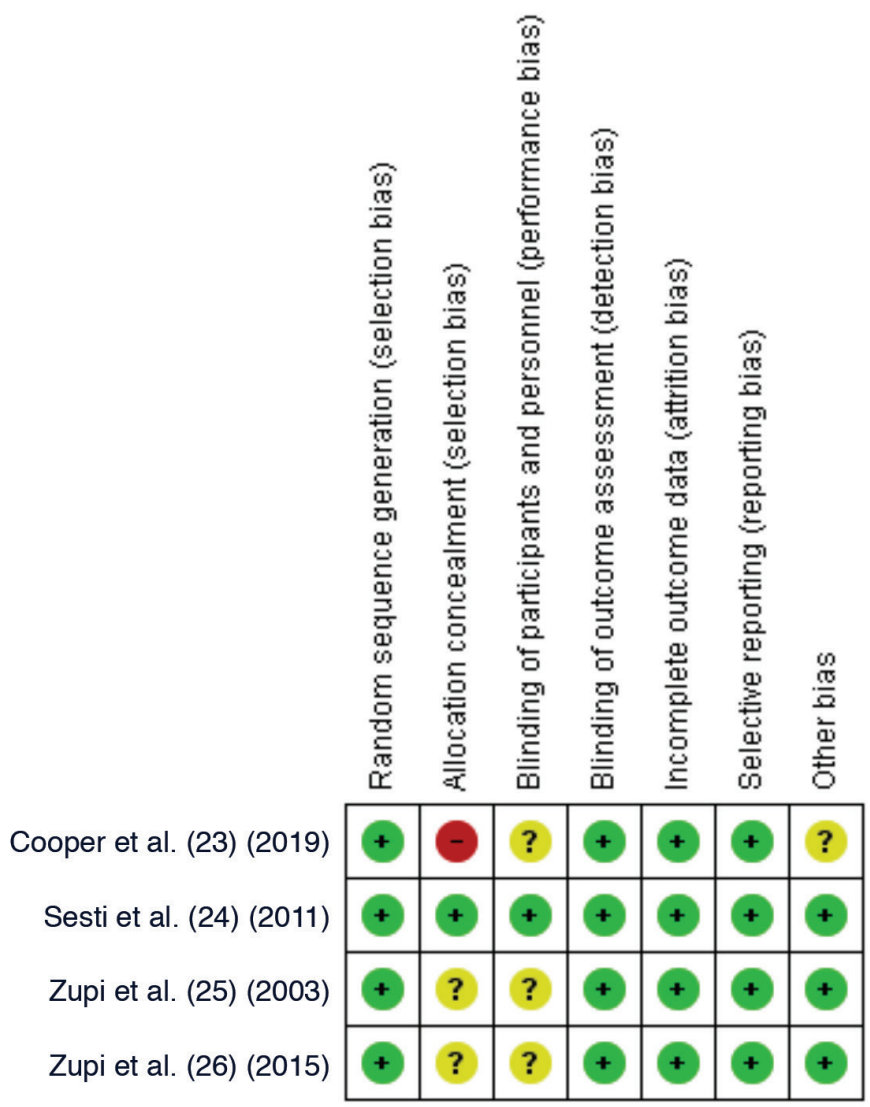

Figure 2. A detailed illustration of the risk of bias of included trials 
function, vitality, and pain). Regarding the general health component, the overall mean difference favored the LSH group significantly [MD: $10.25(7.12,13.38),(\mathrm{p}<0.01)]$. Pooled analysis was homogenous $(\mathrm{p}=0.74) ; \mathrm{I}^{2}: 0 \%$.

As for physical function component, the total mean difference showed no significant difference between both groups [MD: $10.64(-8.08,29.36),(\mathrm{p}=0.27)]$, data was heterogeneous $(\mathrm{p}<0.01) ; \mathrm{I}^{2}: 96 \%$. Concerning the limitation on life functions (physical) component, the combined mean difference showed no significant change between both groups [MD: $1.83(-1.56$, $5.22),(p=0.29)]$. Pooled studies were homogenous $(p=0.41)$; $\mathrm{I}^{2}: 0 \%$.

The analysis of the limitation on life functions (emotional) component yielded no significant variation between both groups [MD: $-7.07 \quad(-28.72,14.59),(p=0.52)]$. Data was heterogeneous $(p<0.01) ; I^{2}: 97 \%$. As for mental health, the comprehensive mean difference showed no significant change between both groups [MD: $-5.33(-21.20,10.55),(p=0.51)]$. The analysis showed heterogeneity $(\mathrm{p}<0.01) ; \mathrm{I} 2: 96 \%$. With regard to the social function component, the total mean difference favored the LSH group significantly [MD: 16.94 (8.32, 25.56), $(p=0.0001)]$. Data was heterogeneous ( $p=0.003) ; \mathrm{I}^{2}: 88 \%$.

Vitality component analysis indicated no significant change between both groups [MD: $0.22(-21.73,22.17),(\mathrm{p}=0.98)]$. The analysis showed heterogeneity $(\mathrm{p}<0.01) ; \mathrm{I}^{2}$ : $98 \%$. The analysis of the pain component showed that there was no significant favoring of either group over the other [MD: $7.67(-4.48,19.83)$, $(\mathrm{p}=0.22)]$. Pooled studies were heterogeneous $(\mathrm{p}=0.0002) ; \mathrm{I}^{2}$ : $93 \%$. Detailed analysis for each item in SF-36 outcome is shown in Figure 4.

Heterogeneity could not be solved in this outcome either by the leave-one-out method or subgroup analysis because only two studies had reported the outcome.

\section{Operation time (in minutes)}

Three studies (23-25) reported operation time. The total mean difference favored the EA group significantly [MD: 72.65 (35.48, 109.82), $(p=0.0001)]$. Pooled studies were heterogeneous $(\mathrm{p}<0.01) ; \mathrm{I}^{2}: 98 \%$ as shown in Figure 5. Heterogeneity could not be solved either by the leave-one-out method or subgroup analysis.

\section{Time from operation to discharge (in Hours)}

Cooper et al. (23) and Zupi et al. (25) reported the time from operation to discharge. The analysis favored the EA group over the LSH group significantly [MD: 13.61 (3.21, 24.01), $(\mathrm{p}=0.01)]$. Data was heterogeneous $(\mathrm{p}=0.02) ; \mathrm{I}^{2}: 81 \%$ as shown in Figure 6 . Heterogeneity could not be solved either by the leave-oneout method or by subgroup analysis.

\section{Hemoglobin (g/dL)}

Sesti et al. (24) and Zupi et al. (25) reported hemoglobin levels postoperatively. Hemoglobin level was significantly lower in the EA group than the LSH group [MD: $0.57(0.40,0.74),(\mathrm{p}<0.01)]$. Pooled analysis was homogenous $(\mathrm{p}=0.22) ; \mathrm{I}^{2}: 34 \%$ as shown in Figure 7.

\section{Pain}

Pain outcome was reported by two studies $(23,25)$. The pain score was assessed by two different scales. Therefore, we performed analysis using a standardized MD (SMD). The overall SMD favored the EA group significantly [SMD: $0.46(0.32,0.60)$, $(\mathrm{p}<0.01)]$. The analysis was homogenous $(\mathrm{p}=0.45) ; \mathrm{I}^{2}: 0 \%$ as shown in Figure 8.

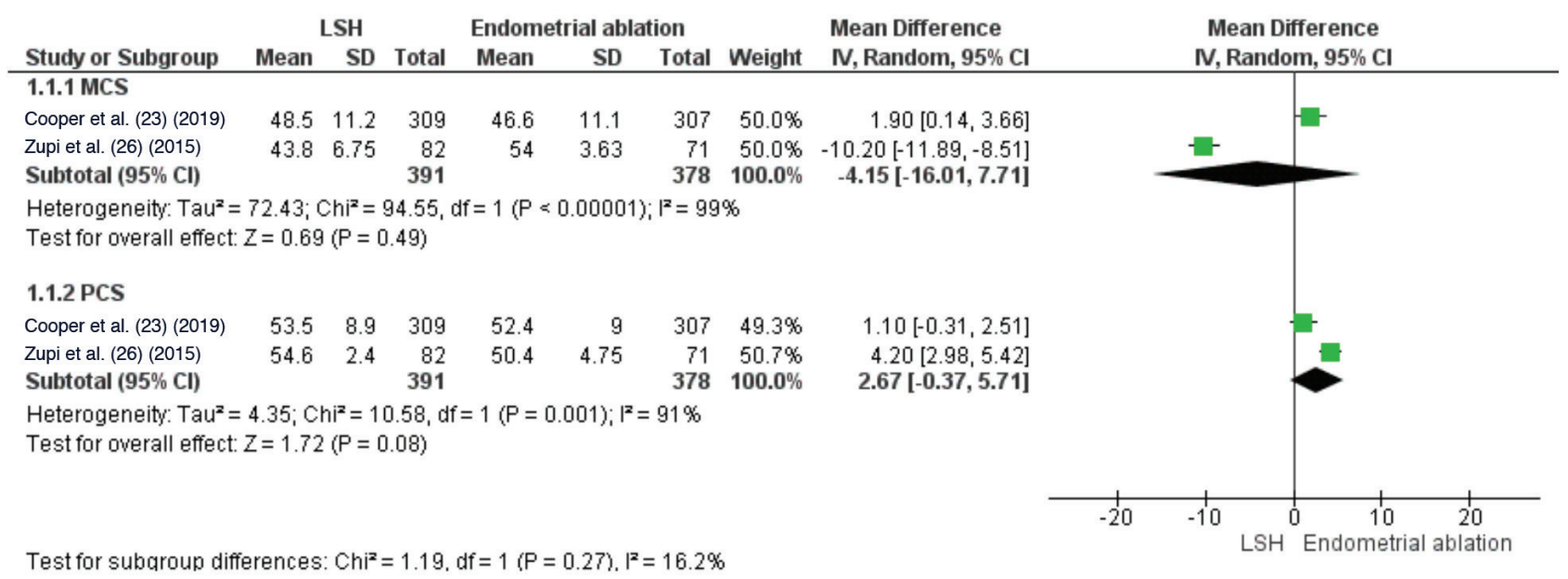

Figure 3. Forest plot for the analysis of SF-12 outcome

LSH: Laparoscopic supracervical hysterectomy, SD: Standard deviation, CI: Confidence interval, MCS: Mental component summary, PCS: Physical component summary 


\section{Fever}

Two studies $(23,25)$ reported fever outcomes postoperatively. The total mean difference showed no significant variation between both groups [MD: $0.00(-0.01,0.02),(\mathrm{p}=0.54)]$. Data was homogenous $(\mathrm{p}=0.76) ; \mathrm{I}^{2}: 0 \%$ as shown in Figure 9.

\section{Discussion}

Some authors have suggested that when limiting comparison to success rates, that surgery has superior results than different oral medications in the treatment of menstrual bleeding in improving the quality of life of women affected by AUB (27). This is not to say, however, that there is complete agreement across all medical and surgical therapies. For example, Madhu et al. (28), found that there was no significant difference between thermal balloon ablation (TBA) as a conservative surgery and use of a levonorgestrel intrauterine system. While hysterectomy is the only curative treatment that prevents menstrual bleeding $100 \%$ in all cases, it also carries a high risk

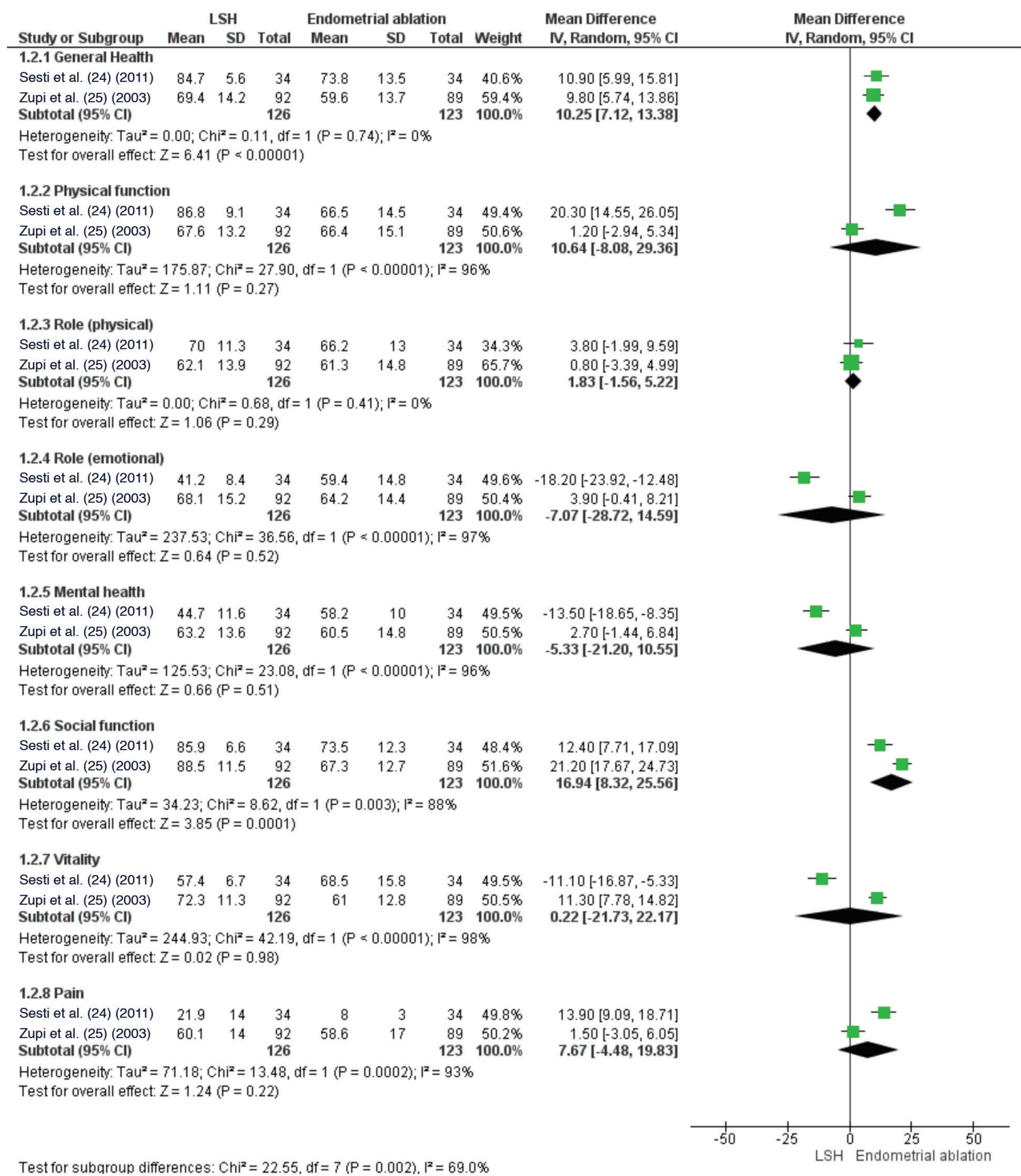

Figure 4. Forest plot for the analysis of SF-36 outcome LSH: Laparoscopic supracervical hysterectomy, SD: Standard deviation, CI: Confidence interval 
for serious complications. EA has been suggested to be the second line of treatment after failure of medical treatment, rather than a hysterectomy, by several authors (29). The best surgical treatment for AUB, therefore, remains controversial. In this systematic review and meta-analysis, the aim was to identify the optimal surgical intervention for the treatment of AUB by comparing the outcomes of only LSH and EA or resection. We chose to compare LSH to EA instead of comparing total laparoscopic hysterectomy (TLH) or laparoscopic assisted vaginal hysterectomy (LAVH) because many authors feel LSH is more minimally invasive than TLH and $\mathrm{LAVH}$, because the surgery does not include the creation of a colpotomy or division of the utero-sacral ligaments. Therefore we aimed to compare two very minimally invasive procedures.

In terms of quality of life, it was found that LSH was significantly superior to EA in general health and in social function. However, in other components of the SF-36 there was no significant difference between LSH and EA. Although the analysis didn't

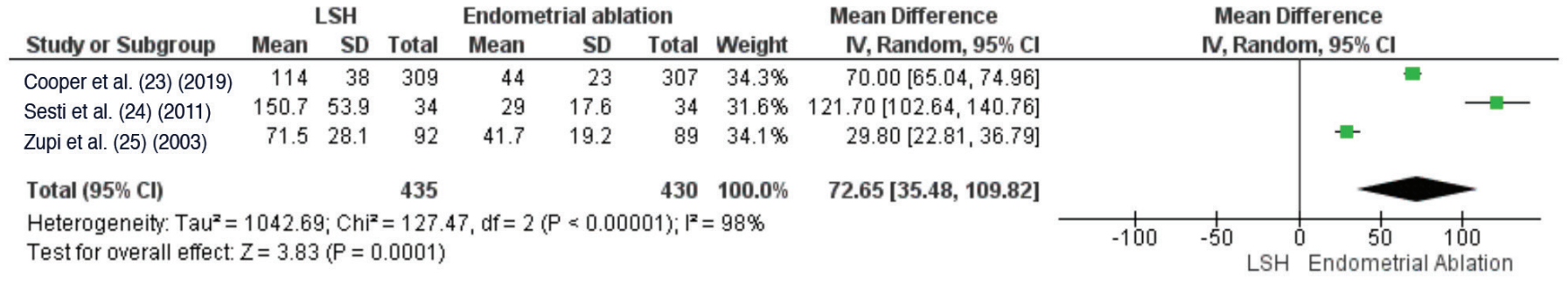

Figure 5. Forest plot for the analysis of operation time outcome LSH: Laparoscopic supracervical hysterectomy, SD: Standard deviation, CI: Confidence interval

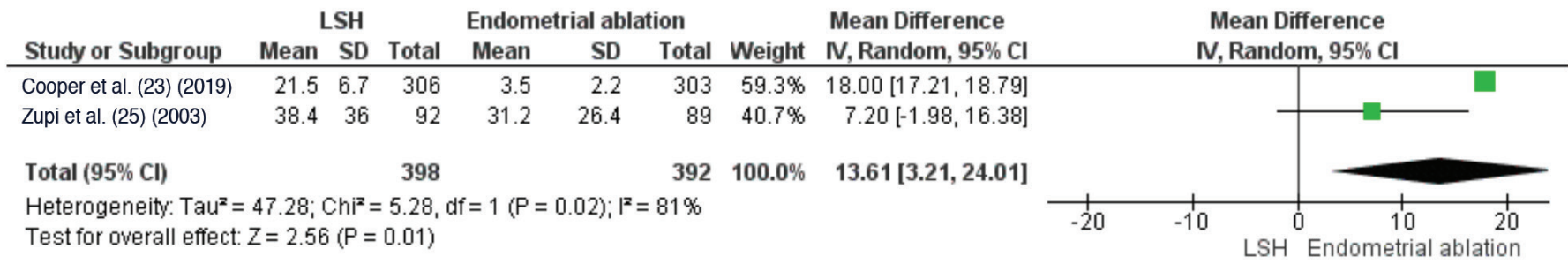

Figure 6. Forest plot for the analysis of time from operation to discharge outcome LSH: Laparoscopic supracervical hysterectomy, SD: Standard deviation, CI: Confidence interval

\begin{tabular}{lrrrrrrrrr} 
& \multicolumn{3}{c}{ LSH } & \multicolumn{3}{c}{ Endometrial ablation } & \multicolumn{3}{c}{ Mean Difference } \\
Study or Subgroup & Mean & SD & Total & Mean & SD & Total & Weight & N, Fixed, 95\% Cl & Mean Difference \\
\hline Sesti et al. (24) (2011) & 14.4 & 1.1 & 309 & 13.8 & 1.1 & 307 & $92.6 \%$ & $0.60[0.43,0.77]$ \\
Zupi et al. (25) (2003) & 12.1 & 2 & 92 & 11.9 & 2.2 & 89 & $7.4 \%$ & $0.20[-0.41,0.81]$ \\
\hline
\end{tabular}

Figure 7. Forest plot for the analysis of hemoglobin outcome LSH: Laparoscopic supracervical hysterectomy, SD: Standard deviation, CI: Confidence interval

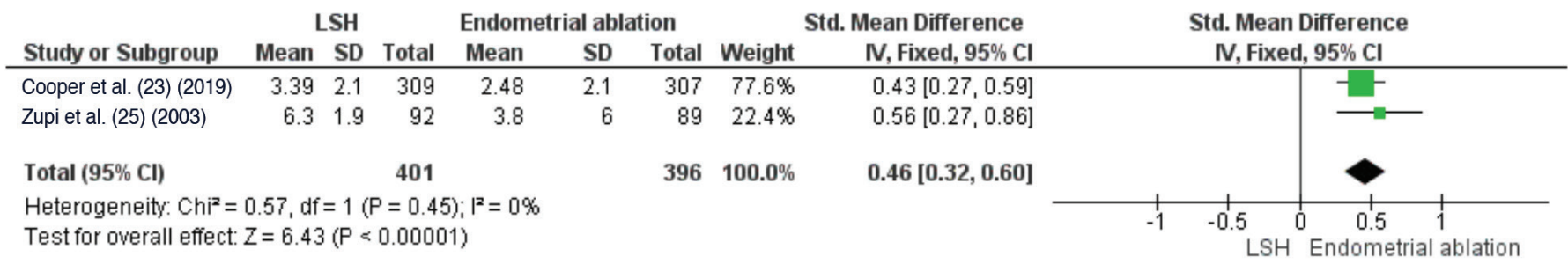

Figure 8. Forest plot for the analysis of pain outcome

LSH: Laparoscopic supracervical hysterectomy, SD: Standard deviation, CI: Confidence interval 


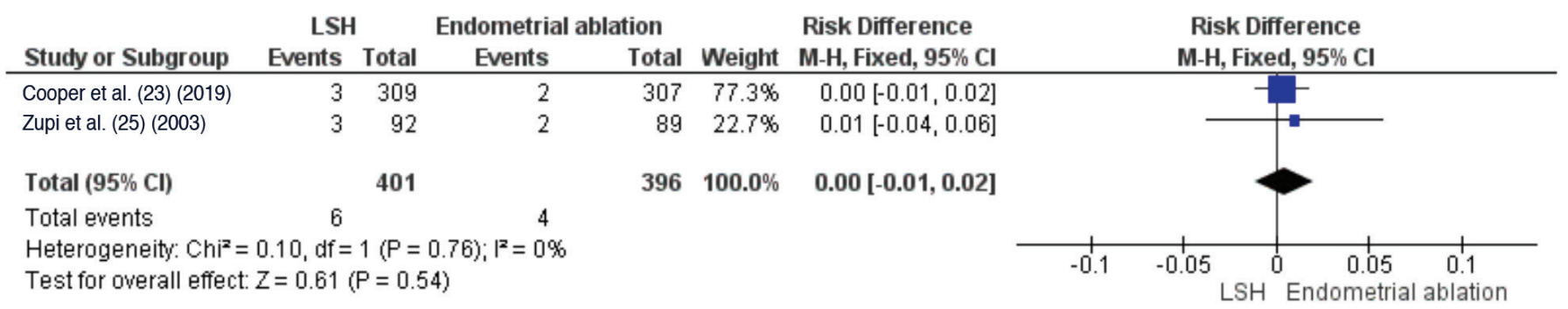

Figure 9. Forest plot for the analysis of fever outcome LSH: Laparoscopic supracervical hysterectomy, SD: Standard deviation, CI: Confidence interval

show a significant difference between the groups in terms of quality of life, except for general health and social function, one study, Cooper et al. (30) reported in a later paper higher satisfaction and quality of life in the LSH group than the EA group at 15 months of randomization. In fact, this study showed there was only $3 \%$ dissatisfaction with LSH compared to a $13 \%$ dissatisfaction rate reported in the EA group. Moreover, 69\% of women in the LSH group reported a maximum state of quality of life with a quality of life percentage score of 100 , compared to only $55 \%$ of women who reported a $100 \%$ score in the EA group. Zupi et al. (31), 2003 showed similar results on their 2-year followup questionnaire. In contrast, O'Connor et al. (32), reported no significant statistical difference regarding satisfaction in the LSH group and the EA group. The follow-up study by Zupi et al. (33), 2015 also showed a better quality of life regarding physical and mental components after 14 years. In addition, Sesti et al. (34) reported a worsening of quality of life in the EA group between 12 months and two years. This is consistent with other nonrandomized trials which reported improvement of psychological outcomes and quality of life after hysterectomy $(35,36)$.

LSH was significantly better than EA regarding postoperative hemoglobin level, which usually reflects the overall amount of blood loss in each group in the three months following the operation. This was in spite of statistically significant longer operation time and a longer duration of hospital stay in the LSH group compared to the EA group. In a later analysis, Cooper et al. (30) showed similar results. The only reasonable explanation for this relative drop of hemoglobin level in the EA group, is that the intact uterus must in some cases still be producing cyclic menstrual bleeding despite the surgery, and that the LSH group was likely no longer menstruating in any reasonable capacity. Other authors, however, have reported that TBA caused a significant decrease in menstrual bleeding and in some cases better hemoglobin levels postoperatively (37). Middleton et al. (38) reported that LSH had adverse impacts on the emotional state of patients. These authors explained their results as coming from the psychological effects of the patients losing their uterus in hysterectomy (38).
Conversely, EA showed significantly lower reported pain compared to LSH in our analysis. This may explain why many women prefer EA to LSH, as it is truly a less invasive procedure, and many women may be afraid of the rare, but possible, major complications that could occur during hysterectomy (39). However, no major complications were reported in our included studies of $\mathrm{LSH}$. This result was also reported in a recent Cochrane study that failed to find any major complications in their reported groups (40). Therefore, we feel it is appropriate to stress that LSH is associated with better improvement in dyspareunia and cyclic pain with no higher risk of postoperative complications (30) than EA by several authors.

\section{Study Limitations}

The main limitations of our study are the paucity of clinical trials that compare the two surgical treatment procedures and the low sample size. Another limitation would be that our analysis was limited to those studies using specific forms in the assessment of the quality of life of the patients receiving the surgical procedures. This could explain, in addition to the different follow-up durations, the great heterogeneity in the analysis of most of our outcomes.

Despite these limitations, this is the first meta-analysis the authors are aware of that compares supracervical hysterectomy as a minimally invasive technique with EA or resection as a more conservative minimally invasive surgical procedure. As for the strong points of our analysis, all the studies included were clinical trials, which helped to ensure the most substantial evidence, as highlighted by the GRADE tool. Another strong point of our analysis was that all the included studies were found to be generally at a low risk of bias using our assessment tools.

\section{Conclusion}

LSH may have better outcomes in regards to quality of life than EA, specifically regarding patient reported social health, patient reported general health, and clinical postoperative hemoglobin 
levels. EA, however, seems to have less operative time and a shorter hospital stay. Therefore, EA is also considered to be a reasonable technique with satisfying outcomes for many women suffering from AUB.

Acknowledgement: The Marchand Institute for Minimally Invasive Surgery would like to acknowledge the efforts of all of the students, researchers, residents and fellows at the institute who put their time and effort into these projects without compensation, only for the betterment of medicine. We firmly assure them that the future of medicine belongs to them.

Ethics Committee Approval: Not applicable to Systematic Review.

Informed Consent: Not applicable to Systematic Review.

Peer-review: Externally peer-reviewed.

Author Contributions: Surgical and Medical Practices: G.J.M.; Concept: A.A., K.S., A.M., S.A.; Design: S.R., K.W., S.H., G.B.; Data Collection or Processing: G.J.M., A.A., K.S., A.M., S.A., S.R., K.W., S.H., G.B., A.K., J.V., K.C., A.G., A.S., J.L.; Analysis or Interpretation: G.J.M., A.A., K.S., A.M., S.A., S.R., K.W., S.H., G.B., A.K., J.V., K.C., A.G., A.S., J.L.; Literature Search: G.J.M., A.A., K.S., A.M.; Writing: A.A., K.S., A.M., S.A., S.R., K.W., S.H., G.B., A.K., J.V., K.C., A.G., A.S., J.L.

Conflict of Interest: No conflict of interest is declared by the authors.

Financial Disclosure: The authors declared that this study received no financial support.

\section{References}

1. Ju H, Jones M, Mishra G. The prevalence and risk factors of dysmenorrhea. Epidemiol Rev 2014; 36: 104-13.

2. National HMB Audit [Internet]. Royal College of Obstetricians \&amp; Gynaecologists. [cited 2020 Sep 23]. Available from: https:// www.rcog.org.uk/en/guidelines-research-services/audit-qualityimprovement/completed-projects/national-HMB-audit/

3. The Implementation and Uptake of Clinical Guidelines in Obstetrics and Gynaecology [Internet]. Royal College of Obstetricians \&amp; Gynaecologists. [cited 2020 Sep 23]. Available from: https:// www.rcog.org.uk/en/guidelines-research-services/audit-qualityimprovement/completed-projects/the-implementation-anduptake-of-clinical-guidelines-in-obstetrics-and-gynaecology/

4. Lethaby A, Farquhar C. Treatments for heavy menstrual bleeding. BMJ 2003; 327: 1243-4.

5. Warner PE, Critchley HO, Lumsden MA, Campbell-Brown M, Douglas A, Menorrhagia I: measured blood loss, clinical features, and outcome in women with heavy periods: a survey with followup data. Am J Obstet Gynecol 2004; 190: 1216-23.

6. Kocaoz S, Cirpan R, Degirmencioglu Az. The prevalence and impacts heavy menstrual bleeding on anemia, fatigue and quality of life in women of reproductive age. Pak J Med Sci 2019; 35: 36570.

7. Stewart EA, Nowak RA. Leiomyoma-related bleeding: a classic hypothesis updated for the molecular era. Hum Reprod Update 1996; 2: 295-306

8. Puri K, Famuyide AO, Erwin PJ, Stewart EA, Laughlin-Tommaso SK. Submucosal fibroids and the relation to heavy menstrual bleeding and anemia. Am J Obstet Gynecol 2014; 210: 38.e1-7.

9. Hapangama DK, Bulmer JN. Pathophysiology of heavy menstrual bleeding. Womens Health (Lond). 2016; 12: 3-13.

10. Marjoribanks J, Lethaby A, Farquhar C. Surgery versus medical therapy for heavy menstrual bleeding. Cochrane Database Syst Rev 2006: CD003855.

11. Overview | Heavy menstrual bleeding: assessment and management | Guidance | NICE [Internet]. NICE; [cited 2020 Sep 26]. Available from: https://www.nice.org.uk/guidance/ng88

12. Fergusson RJ, Lethaby A, Shepperd S, Farquhar C. Endometrial resection and ablation versus hysterectomy for heavy menstrual bleeding. Cochrane Database Syst Rev 2013: CD000329.

13. Nagele F, Rubinger T, Magos A. Why do women choose endometrial ablation rather than hysterectomy? Fertil Steril 1998; 69: 1063-6.

14. Jenkins TR. Laparoscopic supracervical hysterectomy. Am J Obstet Gynecol 2004; 191: 1875-84.

15. Cooper K, Breeman S, Scott NW, Scotland G, Hernández R, Clark $\mathrm{TJ}$, et al. References [Internet]. Laparoscopic supracervical hysterectomy compared with second-generation endometrial ablation for heavy menstrual bleeding: the HEALTH RCT. NIHR Journals Library; 2019 [cited 2020 Sep 26]. Available from: https:// www.ncbi.nlm.nih.gov/books/NBK547196/

16. Persson P, Brynhildsen J, Kjølhede P; Hysterectomy Multicentre Study Group in South-East Sweden. Pelvic organ prolapse after subtotal and total hysterectomy: a long-term follow-up of an open randomised controlled multicentre study. BJOG 2013; 120: 155665.

17. Yang $\mathrm{H}, \mathrm{Xu} \mathrm{X}$, Jiang $\mathrm{X}$, Yao Z. Treatment of menorrhagia due to aplastic anemia by hysteroscopic resection of endometrial functional layer and levonorgestrel-releasing intra-uterine system: Three case reports. Medicine (Baltimore) 2019; 98: e15156.

18. Wouk N, Helton M. Abnormal Uterine Bleeding in Premenopausal Women. Am Fam Physician 2019; 99: 435-43.

19. Ewies AAA, Cooper K, Lee AJ, Chien P, Raja EA, Timmaraju V, et al. Outcomes following hysterectomy or endometrial ablation for heavy menstrual bleeding: retrospective analysis of hospital episode statistics in Scotland reply. Br J Obstet Gynaecol [Internet]. 2012 Feb [cited 2020 Sep 26];119(3). Available from: https:// abdn.pure.elsevier.com/en/publications/outcomes-followinghysterectomy-or-endometrial-ablation-for-heavy

20. Moher D, Liberati A, Tetzlaff J, Altman DG; PRISMA Group. Preferred reporting items for systematic reviews and meta-analyses: the PRISMA statement. PLoS Med 2009; 6: e1000097.

21. Higgins JP, Green S. Cochrane Handbook for Systematic Reviews of Interventions: Cochrane Book Series. :674.

22. Higgins JPT, Altman DG, Gøtzsche PC, Jüni P, Moher D, Oxman AD, et al; Cochrane Bias Methods Group; Cochrane Statistical Methods Group. The Cochrane Collaboration's tool for assessing risk of bias in randomised trials. BMJ 2011; 343: d5928.

23. Cooper K, Breeman S, Scott NW, Scotland G, Clark J, Hawe J, et al; HEALTH Study Group. Laparoscopic supracervical hysterectomy versus endometrial ablation for women with heavy menstrual bleeding (HEALTH): a parallel-group, open-label, randomised controlled trial. Lancet. 2019; 394: 1425-36.

24. Sesti F, Ruggeri V, Pietropolli A, Piancatelli R, Piccione E. Thermal balloon ablation versus laparoscopic supracervical hysterectomy 
for the surgical treatment of heavy menstrual bleeding: a randomized study. J Obstet Gynaecol Res 2011; 37: 1650-7.

25. Zupi E, Zullo F, Marconi D, Sbracia M, Pellicano M, Solima E, et al. Hysteroscopic endometrial resection versus laparoscopic supracervical hysterectomy for menorrhagia: a prospective randomized trial. Am J Obstet Gynecol 2003; 188: 7-12.

26. Zupi E, Centini G, Lazzeri L, Finco A, Zullo F, Exacoustos C. Hysteroscopic Endometrial Resection Versus Laparoscopic Supracervical Hysterectomy for Abnormal Uterine Bleeding: Long Term Follow-Up of a Prospective Randomized Trial. J Minim Invasive Gynecol 2015; 22: S39.

27. Marjoribanks J, Lethaby A, Farquhar C. Surgery versus medical therapy for heavy menstrual bleeding. Cochrane Database Syst Rev 2006; CD003855.

28. Madhu CK, Nattey J, Naeem T. Second generation endometrial ablation techniques: an audit of clinical practice. Arch Gynecol Obstet 2009; 280: 599-602.

29. League DD. Endometrial ablation as an alternative to hysterectomy. AORN J 2003; 77: 322-4.

30. Cooper K, Breeman S, Scott NW, Scotland G, Clark J, Hawe J, et al; HEALTH Study Group. Laparoscopic supracervical hysterectomy versus endometrial ablation for women with heavy menstrual bleeding (HEALTH): a parallel-group, open-label, randomised controlled trial. Lancet 2019; 394: 1425-36.

31. Zupi E, Zullo F, Marconi D, Sbracia M, Pellicano M, Solima E, et al. Hysteroscopic endometrial resection versus laparoscopic supracervical hysterectomy for menorrhagia: a prospective randomized trial. Am J Obstet Gynecol 2003; 188: 7-12.

32. O'Connor H, Broadbent JA, Magos AL, McPherson K. Medical Research Council randomised trial of endometrial resection versus hysterectomy in management of menorrhagia. Lancet 1997; 349: 897-901.
33. Zupi E, Centini G, Lazzeri L, Finco A, Exacoustos C, Afors K, et al. Hysteroscopic Endometrial Resection Versus Laparoscopic Supracervical Hysterectomy for Abnormal Uterine Bleeding: Longterm Follow-up of a Randomized Trial. J Minim Invasive Gynecol $2015 ; 22: 841-5$.

34. Sesti F, Ruggeri V, Pietropolli A, Piancatelli R, Piccione E. Thermal balloon ablation versus laparoscopic supracervical hysterectomy for the surgical treatment of heavy menstrual bleeding: a randomized study. J Obstet Gynaecol Res 2011; 37: 1650-7.

35. Persson P, Wijma K, Hammar M, Kjølhede P. Psychological wellbeing after laparoscopic and abdominal hysterectomy-a randomised controlled multicentre study. BJOG. 2006; 113: 1023-30.

36. Thakar R, Ayers S, Georgakapolou A, Clarkson P, Stanton S, Manyonda I. Hysterectomy improves quality of life and decreases psychiatric symptoms: a prospective and randomised comparison of total versus subtotal hysterectomy. 2004; 111: 1115-20.

37. Iavazzo C, Salakos N, Bakalianou K, Vitoratos N, Vorgias G, Liapis A. Thermal balloon endometrial ablation: a systematic review. Arch Gynecol Obstet 2008; 277: 99-108.

38. Middleton LJ, Champaneria R, Daniels JP, Bhattacharya S, Cooper $\mathrm{KG}$, Hilken $\mathrm{NH}$, et al. Hysterectomy, endometrial destruction, and levonorgestrel releasing intrauterine system (Mirena) for heavy menstrual bleeding: systematic review and meta-analysis of data from individual patients. BMJ (Online). 2010; 341: c3929.

39. Nagele F, Rubinger T, Magos A. Why do women choose endometrial ablation rather than hysterectomy? Fertil Steril 1998; 69: 1063-6.

40. Lethaby A, Mukhopadhyay A, Naik R. Total versus subtotal hysterectomy for benign gynaecological conditions. Cochrane Database Syst Rev 2012; CD004993. 